\title{
THREE-DIMENSIONAL NONLINEAR BEHAVIOUR OF MASONRY WALLS MODELLED WITH DISCRETE ELEMENTS
}

\author{
D. Baraldi ${ }^{1}$ and A. Cecchi ${ }^{2}$ \\ Department of Architecture, Construction and Conservation, Università IUAV di Venezia \\ Dorsoduro 2206, 30123, Venezia, Italy \\ 1e-mail: danielebaraldi@iuav.it \\ 2e-mail: cecchi@iuav.it
}

Keywords: Masonry walls, discrete element model, 3D model, frictional contact, MohrCoulomb yield criterion.

\begin{abstract}
The analysis of masonry structures is of particular interest in the civil engineering and architecture community due to the large amount of historical masonry constructions in Europe and in Italy in particular. Masonry is a heterogeneous material obtained by composition of blocks connected by dry or mortar joints. The use of refined models for investigating the in and out of plane nonlinear behaviour of masonry is an active field of research. Considering historical masonry, the mechanical properties of joints are usually lower than those of blocks, allowing to assume that damage occurs more frequently along joints. For this reason, discrete element models (DEMs) may be frequently adopted for representing masonry behaviour, assuming blocks as rigid bodies and joints as interfaces, with a small number of degrees of freedom and parameters involved in the analysis. As well known, masonry walls may be considered as the most important category of load-bearing elements in masonry structures and they are subject to vertical and horizontal actions generated by gravitational loads and seismic actions, respectively. Horizontal loads may act in plane or out of plane, causing two different collapse mechanisms for a masonry wall. In this work a simple and effective discrete element model, already developed in linear field for in and out of plane analysis and already extended in nonlinear field but only for in plane analysis, is here extended to the three dimensional nonlinear analysis of masonry walls. A Mohr-Coulomb yield criterion is adopted for modelling interface behaviour. A numerical experimentation is carried on in order to determine the limit load multiplier, together with the collapse behaviour, of several masonry walls. Moreover, existing results are taken in consideration in order to calibrate the proposed model and to evaluate its effectiveness.
\end{abstract}




\section{INTRODUCTION}

Masonry is a heterogeneous material composed by natural or artificial blocks connected by dry or mortar joints. Masonry was the main building material for many centuries; earthquake actions are one of the main causes of collapse and the assessment of seismic performance of masonry structures remains a challenging task in the structural analysis field of research.

The strength of masonry structures is affected by geometrical configuration and constructive details. As well known, such details have effects on the two main different seismic induced damages and collapse modes of masonry walls: in-plane and out-of-plane collapse mechanisms. The former are responsible for extended shear deformations and cracks, while the latter may cause the tilting of entire wall portions, leading eventually to the partial or total collapse of the construction. These collapse mechanisms were observed in past earthquakes and adequate descriptions by means of numerical and analytical models were done and are still being developed [1,2].

Several types of numerical models may be adopted for studying masonry behaviour such as heterogeneous or homogenized Finite Element Models (FEMs) or Discrete Element Models (DEMs); moreover, several analysis types may be performed such as limit analysis or incremental analysis. For instance, an overview of the methods for modelling masonry structures may be found in the work of Smoljanović et al. [3] and a deep literature review of out of plane behaviour and models for masonry has been recently done by Ferreira et al. [4].

A DE Model, based on the assumptions of rigid blocks and joints modelled as interfaces, may be suitable for investigating masonry behaviour due to the small number of degrees of freedom (DOFs) needed for performing a numerical analysis of block assemblages. These assumptions may be suitable for historical masonry, in which block stiffness is larger than joint stiffness, allowing to assume blocks as rigid bodies; moreover joint thickness is negligible if compared with block size, especially in case of dry joints, allowing to model joints as interfaces. Discrete models were adopted in the past by many authors for studying masonry out of plane behaviour in linear and nonlinear fields [5-10]. In particular, Cecchi and Sab [7] defined a simple and effective DEM for studying the three-dimensional behaviour of masonry panels with a small computational effort due to the small number of degrees of freedom involved.

In this contribution, the original three-dimensional discrete element model (3D DEM) introduced by Cecchi and Sab [7] in the linear elastic field, is extended to the field of collapse analysis by assuming a Mohr-Coulomb yield criterion for restraining interface actions. With this aim, a nonlinear -elastic perfectly plastic- interface behaviour is considered by assuming action restraints as elastic limits. The theoretical work of Orduña and Lourenço [8] is taken as reference for defining restraint conditions relative to interface shear force, torsion and their combination. Moreover, a static solution approach characterized by the determination of the stiffness matrix of the masonry assemblage is adopted, following and extending the procedure already adopted by authors for the nonlinear incremental analysis of masonry walls in plane loaded [11] and for the in and out of plane modal analysis of masonry walls [12,13]. Several numerical experiments are carried on in order to validate the proposed nonlinear DEM with respect to existing numerical and laboratory results. For first, numeric out of plane tests performed by Orduña and Lourenço [9] are reproduced, then, the experimental campaign on scaled masonry-like specimens performed by Restrepo Vélez et al. [14] is taken in consideration and several cases are reproduced with the proposed nonlinear DEM. In general, the proposed numerical solution method turns out to be effective for the determination of limit loads and out of plane collapse mechanisms of the masonry walls considered. 


\section{THREE DIMENSIONAL DISCRETE MODEL}

A regular masonry with standard running bond periodic pattern is considered; the generic block $B_{i, j}$ is in contact with six neighbours by means of six interfaces or joints $S_{k_{1}, k_{2}}$, with $k_{1}$, $k_{2}= \pm 1$ for horizontal interfaces and $k_{1}= \pm 2, k_{2}=0$, for vertical interfaces (Figure 1). Block dimensions are: $a$ (height), $b$ (width) and $s$ (thickness).

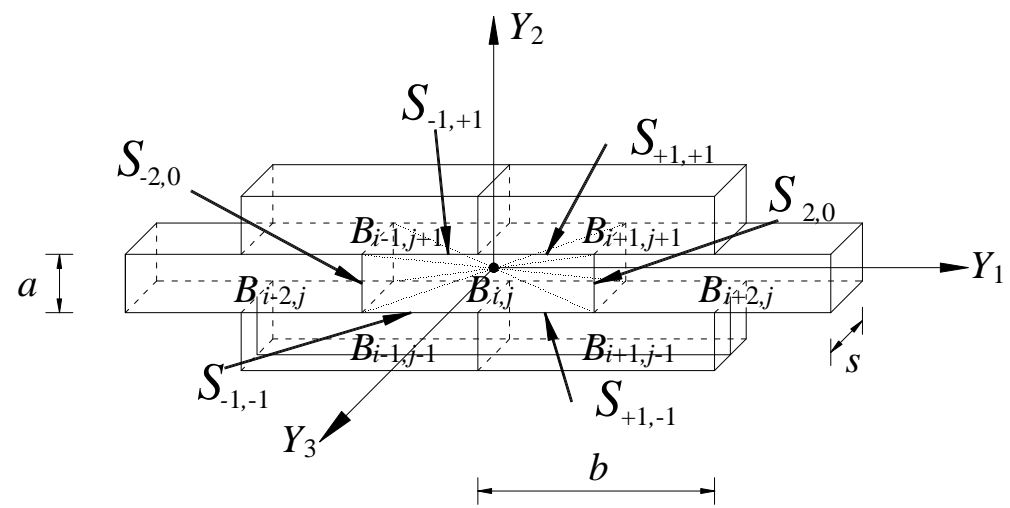

Figure 1. Discrete model, running bond Representative Elementary Volume (REV).

Assuming rigid block hypothesis, the displacement of a generic block is represented by a rigid body motion referred to the motion of its centre and the rotation with respect to its centre:

$$
\mathbf{u}^{i, j}(\mathbf{y})=\mathbf{u}^{i, j}+\mathbf{\Omega}^{i, j}\left(\mathbf{y}-\mathbf{y}^{i, j}\right) .
$$

Where $\mathbf{y}^{i, j}$ is the position of block centre in the Euclidean space: $\mathbf{y}^{i, j}=i(b / 2) \mathbf{e}_{1}+j a \mathbf{e}_{2}$; considering the three dimensional case $\mathbf{u}^{i, j}=\left\{u_{1}^{i, j} u_{2}^{i, j} u_{3}^{i, j}\right\}^{T}$ is the translation vector of $B_{i, j}$ and $\boldsymbol{\Omega}^{i, j}$ is the rotation skew tensor collecting block rotations with respect to coordinate axes:

$$
\boldsymbol{\Omega}^{i, j}=\left[\begin{array}{ccc}
0 & -\omega_{3}^{i, j} & \omega_{2}^{i, j} \\
\omega_{3}^{i, j} & 0 & -\omega_{1}^{i, j} \\
-\omega_{2}^{i, j} & \omega_{1}^{i, j} & 0
\end{array}\right]
$$

In and out of plane block translations and rotations may be collected in $\mathbf{q}^{i, j}=\left\{u_{1}^{i, j} u_{2}^{i, j} u_{3}^{i, j} \omega_{1}^{i, j} \omega_{2}^{i, j} \omega_{3}^{i, j}\right\}^{T}$. Following the procedure described in [7], the interactions between two adjacent blocks $B_{i, j}$ and $B_{i+k_{1}, j+k_{2}}$ through a generic interface $S_{k_{1}, k_{2}}$ are represented by interface tensions $\boldsymbol{\sigma}$ that are related to the relative displacement and rotations between adjacent blocks by means of a constitutive relation $\boldsymbol{\sigma n}=\mathbf{K ~ d}$, neglecting for simplicity apex $k_{1}, k_{2}$. Here $\boldsymbol{\sigma}$ is the stress tensor, $\mathbf{n}$ is the normal vector to the generic interface, $\mathbf{K}$ is the interface stiffness matrix and $\mathbf{d}$ is the vector that collects interface relative translations:

$$
\begin{aligned}
& d_{1}^{k_{1}, k_{2}}=u_{1}^{i+k_{1}, j+k_{2}}-u_{1}^{i, j}+k_{2} a\left(\omega_{3}^{i+k_{1}, j+k_{2}}+\omega_{3}^{i, j}\right) / 2, \\
& d_{3}^{k_{1}, k_{2}}=u_{3}^{i+k_{1}, j+k_{2}}-u_{3}^{i, j}-k_{1}(b / 2)\left(\omega_{3}^{i+k_{1}, j+k_{2}}+\omega_{3}^{i, j}\right) / 2, \\
& d_{3}^{k_{1}, k_{2}}=u_{3}^{i+k_{1}, j+k_{2}}-u_{3}^{i, j}+k_{1}(b / 2)\left(\omega_{2}^{i+k_{1}, j+k_{2}}+\omega_{2}^{i, j}\right) / 2-k_{2} a\left(\omega_{1}^{i+k_{1}, j+k_{2}}+\omega_{1}^{i, j}\right) / 2,
\end{aligned}
$$

and relative rotations: 


$$
\begin{aligned}
& \delta_{1}^{k_{1}, k_{2}}=\omega_{1}^{i+k_{1}, j+k_{2}}-\omega_{1}^{i, j}, \\
& \delta_{2}^{k_{1}, k_{2}}=\omega_{2}^{i+k_{1}, j+k_{2}}-\omega_{2}^{i, j}, \\
& \delta_{3}^{k_{1}, k_{2}}=\omega_{3}^{i+k_{1}, j+k_{2}}-\omega_{3}^{i, j} .
\end{aligned}
$$

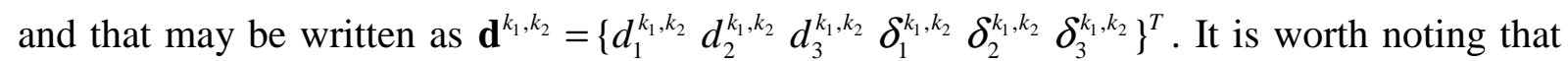
in order to better highlight the relation between relative displacements and global block translations and rotations, the above expressions may be written in matrix form as $\mathbf{d}^{k_{1}, k_{2}}=\mathbf{H}^{k_{1}, k_{2}} \mathbf{q}^{k_{1}, k_{2}}$, where $\mathbf{q}^{k_{1}, k_{2}}$ collects translations and rotations of two adjacent blocks and $\mathbf{H}^{k_{1}, k_{2}}$ may be defined as interface 'compatibility' matrix [15] that collects, following Equations (3)-(4), the relative distances between the centres of the blocks considered.

Assuming initially the hypothesis of elastic interface behaviour, the interface stiffness matrix $\mathbf{K}$ may be detailed for horizontal and vertical case: $\mathbf{K}_{h}^{k_{1}, k_{2}}=\operatorname{diag}\left\{K_{t} K_{n} K_{t} K_{r} K_{t} K_{r}\right\}$ and $\mathbf{K}_{v}^{k_{1}, k_{2}}=\operatorname{diag}\left\{K_{n} K_{t} K_{t} K_{t} K_{r} K_{r}\right\}$, collecting tangential $\left(K_{t}\right)$, normal $\left(K_{n}\right)$ and rotational $\left(K_{r}\right)$ stiffness of the interface. It is worth noting that rotational stiffness assumes the same value of normal stiffness $\left(K_{r}=K_{n}\right)$, but it is defined by a different variable given that in the following description of interface nonlinear behaviour it will be necessary to distinguish between nonlinear behaviour of normal forces with respect to that of bending moments. Assuming mortar joints with an isotropic and elastic behaviour, interfacial stiffness values are function of mortar elastic modulus $E^{m}$ and Poisson ratio $v^{m}[7]$.

The elastic energy of the interface is determined by defining the product of interface stresses and interface relative displacements:

$$
\Pi_{k_{1}, k_{2}}=\frac{1}{2} \int_{S_{k_{1}, k_{2}}} \boldsymbol{\sigma}^{T} \mathbf{d} d S=\frac{1}{2} \int_{S_{k_{1}, k_{2}}} \mathbf{d}^{T} \mathbf{K} \mathbf{d} d S=\frac{1}{2} \mathbf{d}^{T}(\mathbf{K A}) \mathbf{d}=\frac{1}{2} \mathbf{d}^{T} \overline{\mathbf{K}} \mathbf{d},
$$

where apex $k_{1}, k_{2}$ for vectors and matrices is omitted for simplicity, $\mathbf{A}$ is the generic (diagonal) matrix of area and inertias of the interface, that may be detailed for horizontal and vertical cases: $\mathbf{A}_{h}=\operatorname{diag}\left\{S_{h} S_{h} S_{h} I_{h 1}\left(I_{h 1}+I_{h 3}\right) I_{h 3}\right\}, \mathbf{A}_{v}=\operatorname{diag}\left\{S_{v} S_{v} S_{v}\left(I_{v 2}+I_{v 3}\right) I_{v 2} I_{v 3}\right\}$, with

$$
\begin{aligned}
& S_{h}=b s / 2, \quad I_{h 1}=b s^{3} / 24, \quad I_{h 3}=b^{3} s / 96, \\
& S_{v}=a s, \quad I_{v 2}=a s^{3} / 12, \quad I_{v 3}=a^{3} s / 12 .
\end{aligned}
$$

Interface forces and moments may be obtained by differentiating the expression of interface elastic energy in Equation (5) with respect to each block displacement component. Such unknown forces $f_{1}^{k_{1}, k_{2}}, f_{2}^{k_{1}, k_{2}}, f_{3}^{k_{1}, k_{2}}$ and moments $m_{1}^{k_{1}, k_{2}}, m_{2}^{k_{1}, k_{2}}, m_{3}^{k_{1}, k_{2}}$ may be collected in $\mathbf{f}^{k_{1}, k_{2}}=\left\{f_{1}^{k_{1}, k_{2}} f_{2}^{k_{1}, k_{2}} f_{3}^{k_{1}, k_{2}} m_{1}^{k_{1}, k_{2}} m_{2}^{k_{1}, k_{2}} m_{3}^{k_{1}, k_{2}}\right\}^{T}$ and it can be easily demonstrated that $\mathbf{f}^{k_{1}, k_{2}}=\overline{\mathbf{K}}^{k_{1}, k_{2}} \mathbf{d}^{k_{1}, k_{2}}$.

Extending Equation (5) to the entire masonry assemblage (i.e. masonry panel), the total elastic energy $\Pi$ is obtained and the subsequent equilibrium equation for the assemblage subject to generic in and out of plane actions $\mathbf{F}^{\text {ext }}$ is:

$$
\mathbf{F}^{\text {ext }}=\partial \Pi / \partial \mathbf{q}=\mathbf{K}^{\text {panel }} \mathbf{q},
$$

where $\mathbf{q}$ collects block displacements and rotations of the entire panel. Equation (7) may be solved by adopting a molecular dynamics algorithm [7] or directly by explicitly defining the stiffness matrix of the entire assemblage $\mathbf{K}^{\text {panel }}$, joining together the procedures already proposed by authors [11-13] for the in and out of plane cases, respectively, and used separately in 
the field of in and out of plane modal analysis of masonry panels and in the field of nonlinear analysis of masonry panels in plane loaded. In particular, the determination of panel stiffness matrix is based on the definition of a 'compatibility' matrix [15] $\mathbf{H}^{\text {panel }}$ obtained by assembling matrices $\mathbf{H}^{k_{1}, k_{2}}$ over the panel and that relates relative displacements of the entire panel $\mathbf{d}^{\text {panel }}$ with block displacements and rotations: $\mathbf{d}^{\text {panel }}=\mathbf{H}^{\text {panel }} \mathbf{q}$. Then, panel stiffness matrix may be calculated as: $\mathbf{K}^{\text {panel }}=\left(\mathbf{H}^{\text {panel }}\right)^{T} \overline{\mathbf{K}}^{\text {panel }} \mathbf{H}^{\text {panel }}$, where $\overline{\mathbf{K}}^{\text {panel }}$ is a diagonal matrix collecting interface stiffness values of the entire panel.

\subsection{Yield criterion for interface}

The nonlinear behaviour of interfaces is governed by a Mohr-Coulomb yield criterion, characterized by a cohesion $c$ and a friction ratio $\mu=\tan \varphi$. For instance, dry interfaces cannot support tension and have a negligible cohesion, whereas mortar joints are characterized by a tensile strength $\sigma_{t}$ and a cohesion value that may be determined by means of experimental tests (for instance EN 1052-3:2002). However, both interface types are characterized by a frictional behaviour and an unlimited compressive strength is assumed.

Considering a generic interface and assuming a local coordinate system $y_{1} y_{2} y_{3}$, with plane $y_{1} y_{2}$ coincident with interface mid-plane and $y_{3}$ orthogonal to it. Forces and moments exerted by two adjacent blocks at the interface centre are (Figure 2): normal force $f_{3} \mathbf{e}_{3}=f_{n} \mathbf{e}_{3}$, shear forces $f_{1} \mathbf{e}_{1}, f_{2} \mathbf{e}_{2}$, bending moments $m_{1} \mathbf{e}_{1}, m_{2} \mathbf{e}_{2}$ and torsion $m_{3} \mathbf{e}_{3}$.

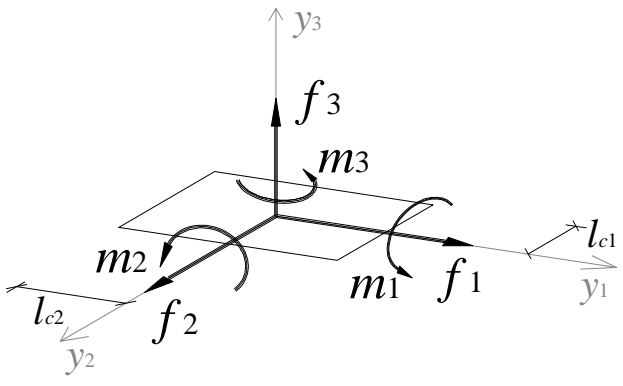

Figure 2: Generic interface with local coordinate system and interface forces and moments.

\subsubsection{Normal and flexural interface strength}

Considering for first interface normal force and bending moments, the nonlinear behaviour is governed by tensile strength and a simple elastic-plastic relation between actions and relative displacements is adopted. Normal force and bending moments must satisfy the following conditions:

$$
\begin{aligned}
& f_{n}=f_{3} \leq f_{t}, \\
& \left|m_{1}\right| \leq\left(f_{t}-f_{n}\right) l_{c 1}, \\
& \left|m_{2}\right| \leq\left(f_{t}-f_{n}\right) l_{c 2} .
\end{aligned}
$$

Where $f_{t}$ represents the tensile strength of the interface, defined as the product of joint tensile strength $\sigma_{t}$ and area of the interface $\left(S_{v}\right.$ or $\left.S_{h}\right)$ and $l_{c i}$ with $i=1,2$ is the characteristic length of the interface with respect to interface plane directions, namely the maximum eccentricity of normal force with respect to block centre that may be supported by the interface. Each value 
is equal to $l_{c 1}=s / 2, l_{c 2}=b / 4$ for a horizontal interface and to $l_{c 1}=a / 2, l_{c 2}=s / 2$ for a vertical one.

First and second conditions in Equation (8) have been already adopted by authors for the in plane case [11] and they turn out to be coincident to those adopted by Trovalusci and Masiani [16]. In particular, the first condition may be defined as 'detachment' condition, whereas the second and third ones may be defined as 'rotation' conditions with respect to $y_{1}$ and $y_{2}$ axis.

The relationship between interface normal force and relative normal displacement and the relationships between interface bending moments and relative rotations follow an elasticperfectly plastic behaviour. In particular, if the interface is subject to excessive moment, only its rotational stiffness is set equal to zero, whereas if the interface is subject to detachment, all interface stiffness values are set equal to zero:

$$
\begin{aligned}
& f_{n}>f_{t} \rightarrow K_{n}=K_{t}=K_{r}=0, \\
& \left|m_{i}\right|>\left(f_{t}-f_{n}\right) l_{c i} \rightarrow K_{r}=0 \quad i=1,2 .
\end{aligned}
$$

\subsubsection{Shear and torsion interface strength}

Differently than the in plane case, characterized by interface shear force acting together with a normal force and a bending moment, in the three dimensional case shear stresses are generated by two components of shear force $f_{1}$ and $f_{2}$ and by a torsion $m_{3}$ and such stresses act together with normal stresses generated by normal force and bending moments. Limit conditions for shear forces and torsion adopted here follow the expressions introduced by Orduña and Lourenco [8]. In particular, if torsion is not present, uniform shear stresses are assumed over the interface and the well-known Mohr-Coulomb yield criterion is applied to the resultant of interface shear forces as follows:

$$
\left|f_{s}\right|=\sqrt{f_{1}^{2}+f_{2}^{2}} \leq\left(f_{t}-f_{n}\right) \tan \varphi \text { if } m_{3}=0 .
$$

The condition above may be defined as 'sliding' condition and the quantity $\left[\left(f_{t}-f_{n}\right) \tan \varphi\right]$ represents the shear strength of the interface. It is worth noting that in this case the cohesion of the interface is represented by the shear strength in absence of a normal action, hence it can be assumed that such strength is equal to $\left[c S_{h}=f_{t} \tan \varphi\right.$ ] for a horizontal interface and to $\left[c S_{v}=f_{t} \tan \varphi\right.$ ] for a vertical one.

If shear forces over the interface are equal to zero, the torsion generates shear stresses depending on relative rotation and on the distance from interface centre, that represents the twisting centre. Torsion over the interface must be less than the torsion strength as follows:

$$
\left|m_{3}\right| \leq C_{t}\left(f_{t}-f_{n}\right) \tan \varphi \text { if } f_{s}=0,
$$

where $C_{t}$ is the torsion constant defined in [8], that is based on an interface subdivision into eight rectangular triangles. If shear forces and torsion act together over the interface, the twisting centre moves away from interface centre and the shear-torsion interaction may be defined analytically by integrating shear stresses over the interface. The simplified sheartorsion interaction curve defined by Orduña and Lourenço [8] is adopted. An elastic-plastic behaviour is assumed also in this case and if restraint conditions for shear force and torsion are not respected, the interface tangential stiffness $K_{t}$ is set equal to zero, whereas normal and flexural stiffness values are not modified.

In order to appreciate the correct adoption of shear and torsion nonlinear behaviour in the proposed DEM, a simple specimen of two blocks connected by an interface modelled with DEM is tested by applying to the interface an increasing torsion with several fixed values of 
shear force and by applying an increasing shear force with several fixed values of torsion. Friction ratio $\mu=0.7$ is assumed and a normal force $f_{n}=240 \mathrm{~N}$ is applied over the interface. Interface size are $0.2 \mathrm{~m} \times 0.3 \mathrm{~m}$. The following Figure 3 shows separately the torsion-relative rotation incremental curves for varying shear force and the shear force-relative displacement incremental curves for varying torsion. As expected, torsion strength decreases for increasing the applied shear force and similarly shear strength decreases for increasing the applied torsion. Both groups of incremental curves allow to obtain the simplified shear-torsion domain proposed by Orduña and Lourenço [8].

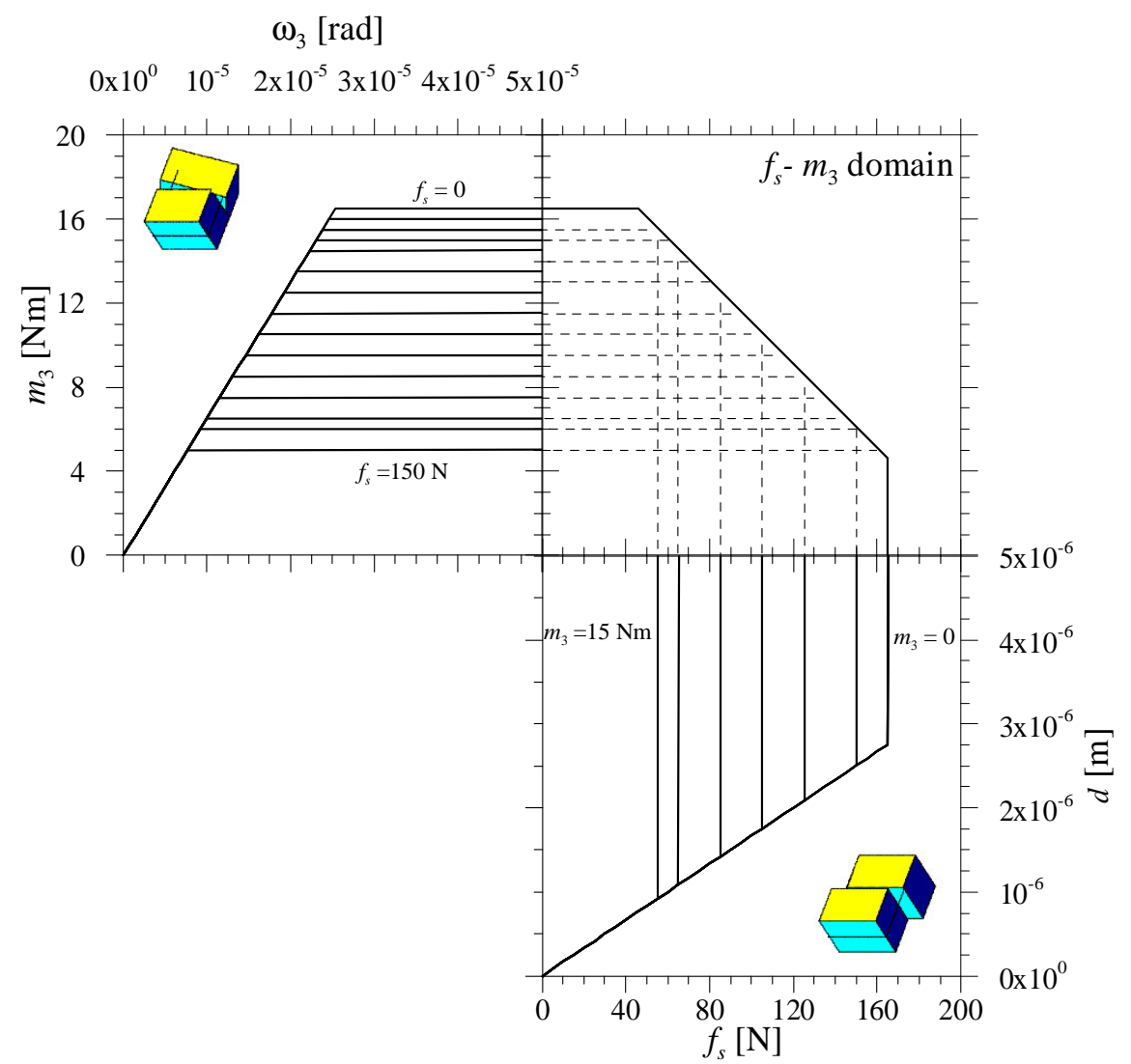

Figure 3: Incremental analyses of a simple specimen subject to increasing torsion with several values of fixed shear force and subject to increasing shear force with several values of torsion.

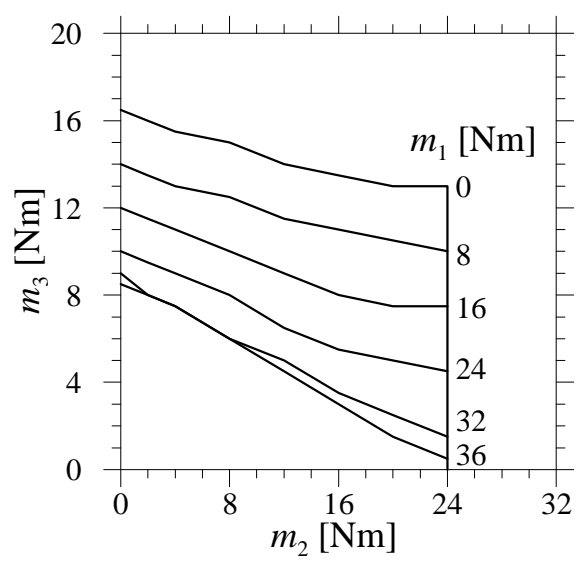

Figure 4: Torsion strength-bending moment interaction curves. 
Finally, if bending moments act over the interface together with shear force and torsion, the effective area of the interface is reduced taking into account eccentricity values $e_{1}=m_{2} / f_{n}$ and $e_{2}=m_{1} / f_{n}$, then the torsion constant $C_{t}$ gets smaller, leading to a smaller torsion strength with respect to the case without bending moments. Considering again a simple specimen made of two blocks connected by an interface and varying both values of bending moments over the interface, Figure 4 shows the corresponding torsion strength values and the curves obtained numerically are in excellent agreement with those obtained analytically by Orduña and Lourenço [8].

\subsection{Nonlinear DEM}

As previously stated, restraint conditions for interface forces and moments allow to define the elastic limits of force-relative displacement and moment-relative rotation constitutive laws, that are assumed to be elastic-perfectly plastic. Then, incremental analyses may be performed by applying incremental load steps to the discrete model and updating the stiffness matrix accounting for local interface nonlinear behaviour. For instance, the stiffness matrix at a generic load step, needed for the evaluation of the corresponding increment of displacements $\delta \mathbf{q}_{i}$ and internal forces $\mathbf{F}_{i}^{i n t}$, is based on interface damage at the previous step as follows: $\delta \mathbf{q}_{i}=\left(\mathbf{K}_{\left(\mathbf{q}_{i-1}\right)}^{\text {panel }}\right)^{-1} \delta \mathbf{F}^{\text {ext }}$ and $\mathbf{F}_{i}^{\text {int }}=\mathbf{F}_{i-1}^{\text {int }}+\mathbf{K}_{\left(\mathbf{q}_{i-1}\right)}^{\text {pane }} \delta \mathbf{q}_{i}$. Then, internal forces are corrected accounting for the yield criterion adopted and residual forces $\mathbf{R}_{i}=\mathbf{F}_{i}^{\text {int }}-\mathbf{F}^{\text {ext }}$ are determined as a starting point of an iterative process of residuals minimization.

In the following paragraphs, numerical tests are performed considering masonry panels subject to self-weight and increasing lateral loads (in or out of plane). Such loads are proportional to the weight ( $P=\gamma a b s$, where $\gamma$ is block volumetric weight) and are identified by the load multiplier $\lambda$; then, the vector collecting the total forces applied at block centres may be defined as $\mathbf{F}^{\text {ext }}=\mathbf{F}_{D}+\lambda \mathbf{F}_{L}$, where $\mathbf{F}_{D}$ represents dead loads, collecting block weight $P$ and $\lambda \mathbf{F}_{L}$ represents unknown horizontal live loads (in or out of plane).

\section{IN PLANE NUMERICAL EXPERIMENTS}

In order to appreciate the effectiveness of the proposed nonlinear DEM, several numerical experiments of masonry panels in plane loaded by self weight and increasing lateral loads are performed. For instance, the numerical tests originally performed by Baggio and Trovalusci [17] and already studied by authors [11] are reproduced, obtaining the same results of previous in plane analyses (Figure 5).
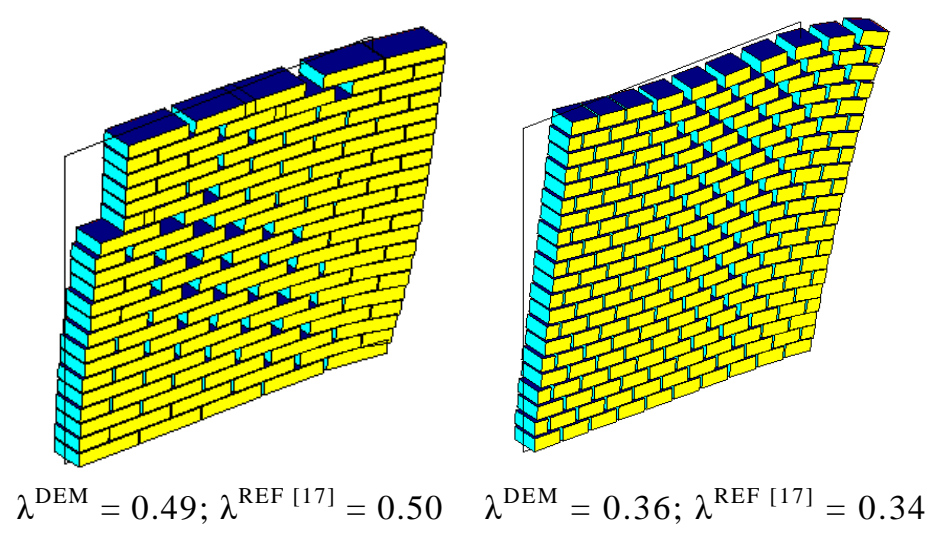

Figure 5: Failure mechanisms for masonry panels subject to self weight and increasing in plane lateral loads. 


\section{OUT OF PLANE NUMERICAL EXPERIMENTS}

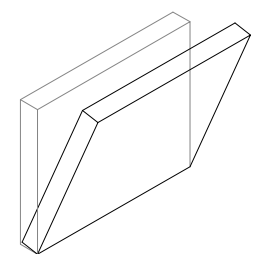

a

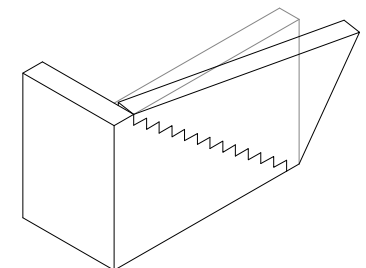

b

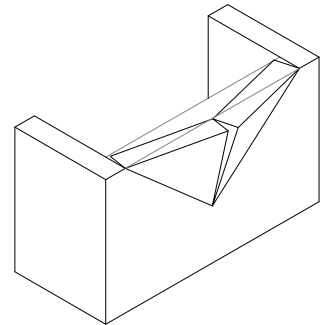

c

Figure 6: Typical out of plane collapse mechanisms for a masonry wall [18].

In this paragraph, several numerical experiments are carried on in order to evaluate the effectiveness of the proposed nonlinear DEM in the determination of out of plane collapse mechanisms and limit loads of masonry panels. In particular, the typical mechanisms described by Rondelet [18] are going to be reproduced numerically and several existing results, both numerical and experimental, are taken as reference. In particular, the first mechanism type defined by Rondelet is the typical out of plane rigid rotation of a free masonry wall with respect to its base (Fig. 6a), whereas the second mechanism regards a masonry wall restrained along a vertical edge by an orthogonal wall, leading to the formation of a diagonal crack and to a roto-translation of a triangular portion of the wall (Fig. 6b). This mechanism is characterized by a larger ultimate load with respect to the previous case. Finally the third mechanism regards a masonry wall restrained by two orthogonal walls at both vertical edges; in this case a vertical crack along panel axis of symmetry forms together with two diagonal cracks starting from panel upper corners (Fig. 6c), leading to two triangular portions that rotate with respect to diagonal cracks. In this last case, the ultimate load is larger than those of the previous mechanisms.

\subsection{Case studies proposed by Orduña and Lourenço}

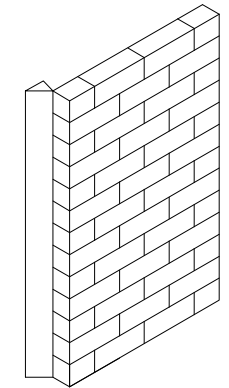

a

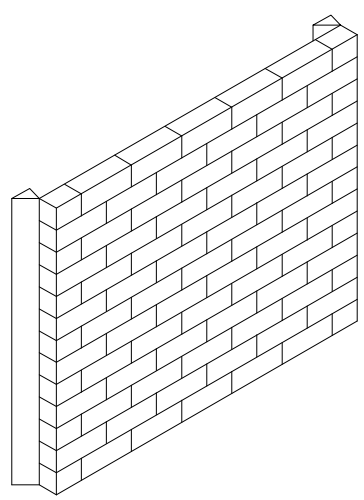

b

Figure 7: Case studies considered originally by Orduña and Lourenço [9].

Two simple examples of masonry panels out of plane loaded are taken into account for first, by assuming as reference the numerical tests performed by Orduña and Lourenço [9]. In both cases masonry panels are made of blocks assembled following a running bond pattern and having dimensions $a=0.081 \mathrm{~m}, b=0.210 \mathrm{~m}, s=0.07 \mathrm{~m}$ and volumetric weight equal to $20 \mathrm{kN} / \mathrm{m}^{3}$. Contacts between blocks are dry, with $\mu=0.7$ and null cohesion. In the first example, panel overall dimensions are: length $L=0.630 \mathrm{~m}$, height $H=1.053 \mathrm{~m}$ and thickness $s=$ 
$0.07 \mathrm{~m}$, obtained by assembling 3 blocks along panel length and 13 blocks along its height; moreover block translations are fixed along panel left column (Figure 7a). In the second example panel dimensions are: $L=1.260 \mathrm{~m}, H=1.053 \mathrm{~m}$ and $s=0.07 \mathrm{~m}$, obtained with 6 blocks along panel length and 13 blocks along its height. In this case, block translations are fixed along external columns (Figure 7b).

Figure 8a shows a collapse mechanism characterized by a diagonal crack starting from the right side of the panel after the 2nd row of blocks. Such mechanism is in quite good agreement with the one showed in the original analysis and the limit load obtained with the proposed nonlinear DEM is included between FEM and limit analysis performed by Orduña and Lourenço (Table 1). Figure 8b shows a collapse mechanism characterized by a symmetric flexural deformation with large displacements along vertical axis of symmetry. Similarly to the previous case, collapse mechanism and limit load (Table 1) are in quite good agreement with reference solutions.
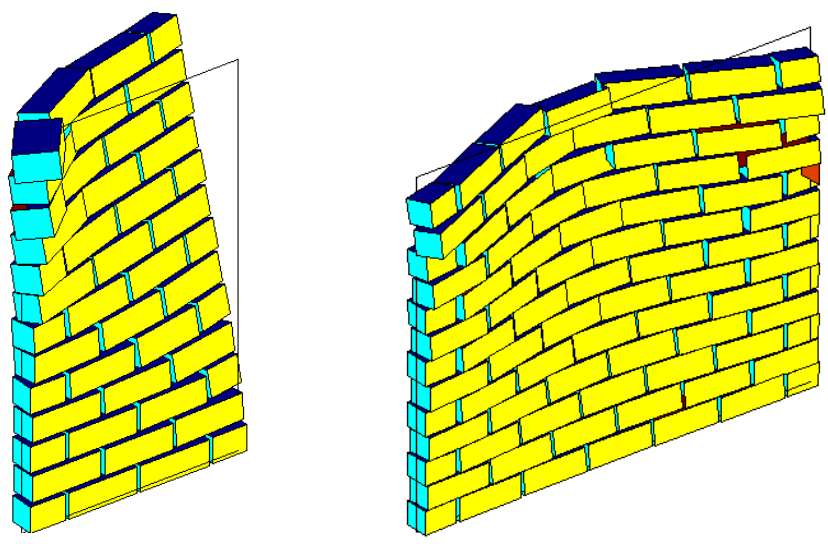

b

Figure 8: Failure mechanisms for the masonry panels studied by Orduña and Lourenço [9] modelled with DEM.

\begin{tabular}{lcc}
\hline & case 1 & case 2 \\
\hline$\lambda^{\text {DEM }}$ & 0.175 & 0.215 \\
$\lambda^{\text {REF,FEM }}$ & 0.210 & 0.260 \\
$\lambda^{\text {REF,lim }}$ & 0.127 & 0.193 \\
\hline
\end{tabular}

Table 1: Limit loads obtained with DEM and reference results of the masonry panels studied by Orduña and Lourenço [9].

\subsection{Restrepo Vélez, Magenes and Griffith experiments}

In this paragraph, several experimental tests performed by Restrepo Vélez et al. [14] are taken as reference. Original tests were performed on scaled masonry-like specimens with dry joints, subject to self-weight and increasing out of plane loads by means of an inclined plane machine, in order to obtain out of plane failure mechanisms. Block dimensions are $a=28.24$ $\mathrm{mm}, b=79.78 \mathrm{~mm}$ and $s=39.68 \mathrm{~mm}$ and block volumetric weight is $26.8 \mathrm{kN} / \mathrm{m}^{3}$. Dry joints are characterized by friction ratio $\mu=0.7$ and null cohesion. All cases are characterized by 21 block courses along panel height and varying number $n$ of blocks along panel length (from 4 to 14, for instance). Panel restraints at one or both lateral edges were obtained with one or two orthogonal walls (Figure 9a and b, respectively); for this reason, several mechanisms turned out to involve also blocks in orthogonal walls. In the present campaign, the effect of orthogo- 
nal walls is simply modelled by restraining block translations and rotations along one or both lateral columns (Figure 9d and e, respectively). Then, the present model will not be able to represent complex collapse mechanisms involving orthogonal walls, leading to small differences between proposed numerical results and experimental tests. Moreover, an additional specimen type considered a panel without orthogonal walls simply supported at the base and loaded by several wooden beams (Figure 9c).

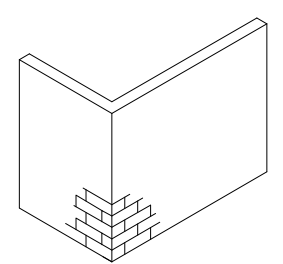

a

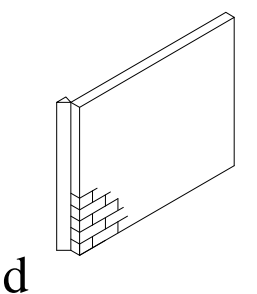

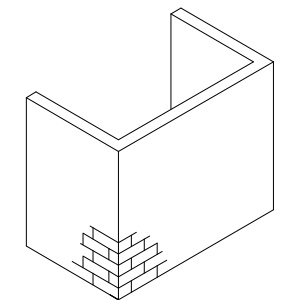

b

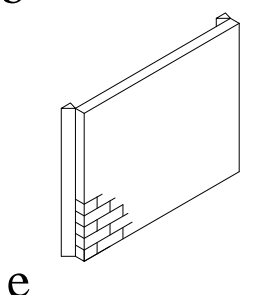

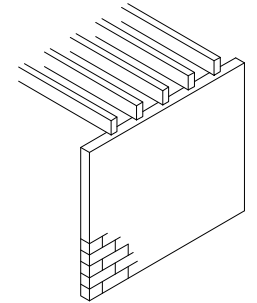

c

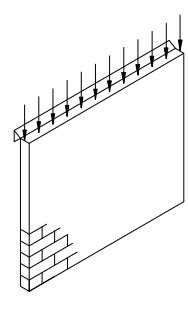

Figure 9: Masonry specimen types considered in the experimental campaign by Restrepo Vélez et al [14] (ac); corresponding specimens modelled with DEM in present analysis (d-f).

Figure 10 shows failure mechanisms of several panels restrained along left column, varying the number of blocks along panel length (for further details about block number see the first row of Table 2). Mechanisms are characterized by a diagonal crack starting from lower-right panel corner, directed towards the upper-left panel corner and a triangular/trapezoidal portion of panel is subject to a roto-translation with respect to such diagonal crack. These mechanism types are in quite good agreement with experimental tests, moreover numerical results obtained with the proposed nonlinear DEM are in quite good agreement with experimental results (Table 2), especially for specimens S11, S12 and S13, thanks to the large number of blocks of the models, whereas for specimen S15, characterized by a small number of blocks along panel length, limit load obtained with DEM is quite far from experimental result with respect to other cases.

Figure 11 shows failure mechanisms of several panels restrained along both lateral vertical edges. Such mechanisms are characterized by a vertical crack along the axis of symmetry of the panel and diagonal cracks starting from lateral edges and moving down to panel axis of symmetry. Mechanisms are similar but not coincident with respect to those obtained experimentally, due to the real restraint adopted for masonry specimens; however limit loads obtained numerically with nonlinear DEM are still in quite good agreement with experimental results.

Finally figure 12 shows the failure mechanism of a simply supported panel loaded by eleven wooden beams (16.46 $\mathrm{N}$ transmitted by each beam) and subject to increasing out of plane loads (see Figure 9c for the corresponding specimen type and Figure 9f for the corresponding DEM representation). The mechanism is characterized by a horizontal hinge in the upper portion of the panel, along the joints between the $15^{\text {th }}$ and $16^{\text {th }}$ block courses and it is almost coincident with the real mechanism obtained during laboratory tests; similarly, the collapse load is close to the one evaluated experimentally. 

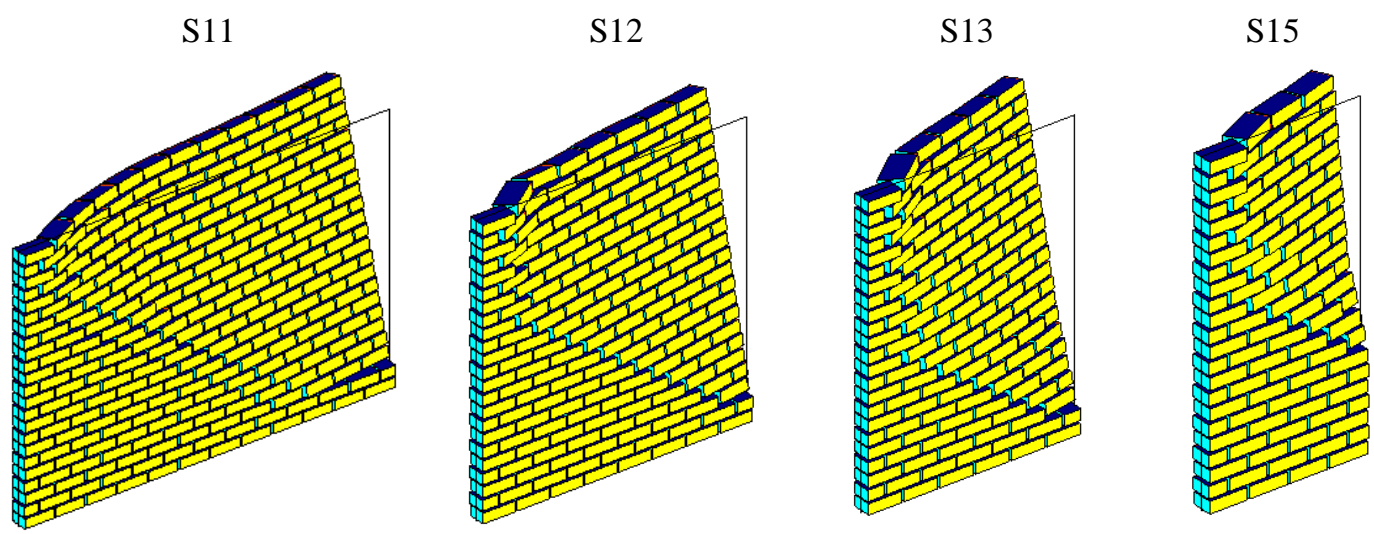

Figure 10: Failure mechanisms for several masonry panels restrained along left column considered by Restrepo Vélez et al. [14] modelled with DEM.
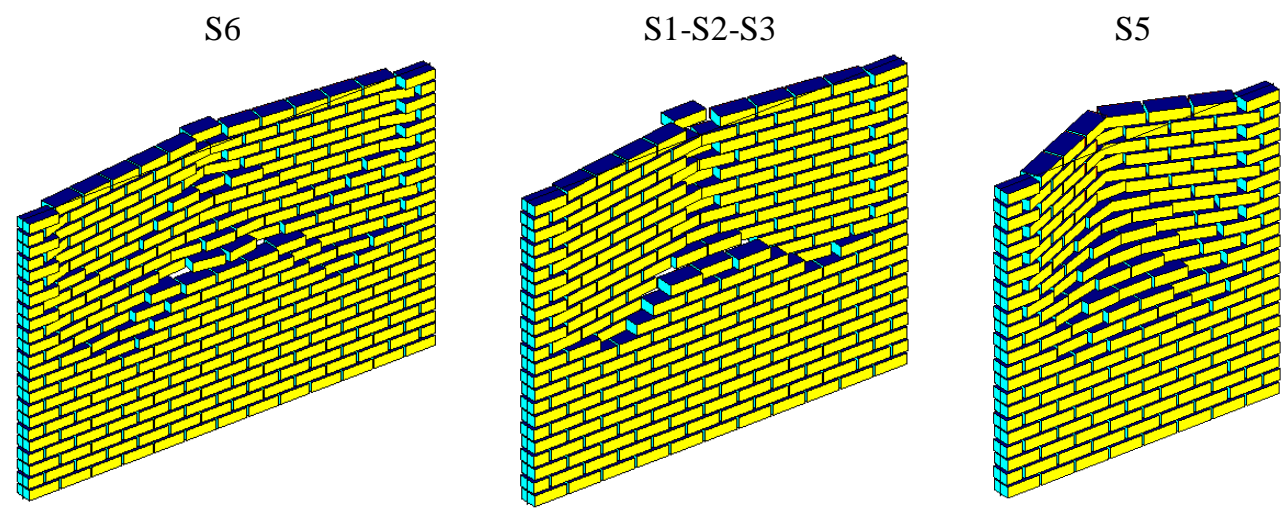

Figure 11: Failure mechanisms for several masonry panels restrained along lateral columns considered by Restrepo Vélez et al. [14] modelled with DEM.

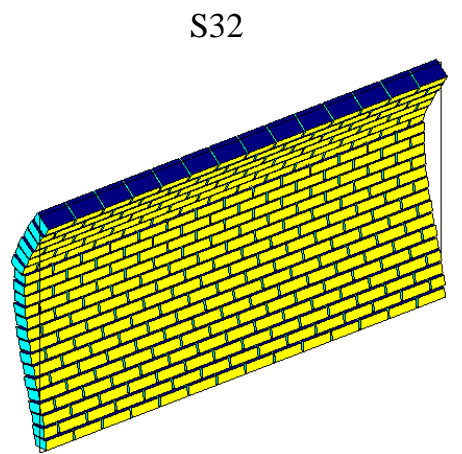

Figure 12: Failure mechanisms for the masonry panel loaded by a set of wooden beams and simply supported at its base considered by Restrepo Vélez et al. [14] modelled with DEM.

\begin{tabular}{rcccccccc}
\hline & S11 & S12 & S13 & S15 & S6 & S1-S2-S3 & S5 & S32 \\
\hline$n$ & 12 & 8 & 6 & 4 & 13 & 11 & 8 & 14 \\
$\lambda^{\text {DEM }}$ & 0.100 & 0.125 & 0.165 & 0.250 & 0.160 & 0.225 & 0.350 & 0.305 \\
$\lambda^{\text {REF }}$ & 0.097 & 0.129 & 0.181 & 0.199 & 0.208 & 0.208 & 0.349 & 0.293 \\
\hline
\end{tabular}

Table 2: Limit loads obtained with DEM and reference results of the masonry specimens considered by Restrepo Vélez et al. [14]. 


\section{CONCLUSIONS}

- In this contribution, the three-dimensional DEM introduced by Cecchi and Sab [7] for modelling regular masonry in the linear elastic field has been extended to the collapse analysis field by adopting a Mohr-Coulomb yield criterion and following the work of Orduña and Lourenço [8] for defining the interaction between interface shear forces, torsion and bending moments.

- The proposed nonlinear DEM for three-dimensional analysis turned out to be simple and effective in the determination of limit loads and collapse mechanisms of masonry panels having regular texture and dry joints, subject to self weight and out of plane loads.

- Several numerical tests reproduced the laboratory campaign carried out by Restrepo Vélez et al. [14], in this case the nonlinear DEM simulated correctly limit loads of the specimens taken into account. Moreover, considering collapse mechanisms, the ones relative to panels restrained by a lateral orthogonal wall have been reproduced correctly by the DEM, whereas small differences have been found in collapse mechanisms relative to panels restrained by orthogonal walls along panel lateral edges.

- Further developments of the model will regard the assessment of nonlinear analysis of more complex masonry specimens, characterized for example by blocks arranged irregularly, openings, lintels and also by real orthogonal walls and roofs.

\section{REFERENCES}

[1] G. de Felice, R. Giannini, Out of plane seismic resistance of masonry walls. Journal of Earthquake Engineering, 5 (2), 253-271, 2001.

[2] D. D'Ayala, E. Speranza, Definition of collapse mechanisms and seismic vulnerability of masonry structures. Earthquake Spectra, 19 (3), 479-509, 2003.

[3] H. Smoljanović, N. Živaljić, Ž. Nikolić, Overview of the methods for the modeling of historical masonry structures. Gradevinar, 65 (7), 607-618, 2013-

[4] T.M. Ferreira, A.A: Costa, A. Costa, Analysis of the Out-Of-Plane Seismic Behavior of Unreinforced Masonry: A Literature Review. International Journal of Architectural Heritage: Conservation, Analysis, and Restoration, 9 (8), 949-972, 2015.

[5] C. Baggio, P. Trovalusci, Limit analysis for no-tension and frictional three-dimensional discrete systems. Mechanics of Structures and Machines, 26 (3), 287-304, 1998.

[6] S. Casolo, Modelling the out-of-plane seismic behaviour of masonry walls by rigid elements. Earthquake Engineering and Structural Dynamics, 29, 1797-1813, 2000.

[7] A. Cecchi, K. Sab, A comparison between a 3D discrete model and two homogenised plate models for periodic elastic brickwork. International Journal of Solids and Structures, 41 (9-10), 2259-2276, 2004.

[8] A. Orduña, P.B. Lourenço, Three-dimensional limit analysis of rigid blocks assemblages. Part I: Torsion failure on frictional interfaces and limit analysis formulation. International Journal of Solids and Structures, 42 (18-19), 5140-5160, 2005.

[9] A. Orduña, P.B. Lourenço, Three-dimensional limit analysis of rigid blocks assemblages. Part II: Load-path following solution procedure and validation. International Journal of Solids and Structures, 42 (18-19), 5161-5180, 2005.

[10] I. Stefanou, J. Sulem, I. Vardoulakis, Three-dimensional Cosserat homogenization of masonry structures: elasticity. Acta Geotechnica, 3, 71-83, 2008. 
[11] D. Baraldi, A. Cecchi, Discrete approaches for the nonlinear analysis of in plane loaded masonry walls: Molecular dynamic and static algorithm solutions. European Journal of Mechanics A/Solids, 57, 165-177, 2016.

[12] D. Baraldi, A. Cecchi, A. Tralli, Discrete and Continuous Models for Out-of-Plane Loaded Masonry Like Structures: A Multiscale Comparative Study. J. Kruis, Y. Tsompanakis, B.H.V. Topping, (Editors), Proceedings of the Fifteenth International Conference on Civil, Structural and Environmental Engineering Computing, Civil-Comp Press, Stirlingshire, UK, Paper 207, Prague, Czech Republic, September 1-4, 2015.

[13] D. Baraldi, S. Bullo, A. Cecchi, Continuous and discrete strategies for the modal analysis of regular masonry. International Journal of Solids and Structures, 84, 82-98, 2016.

[14] L.F. Restrepo Vélez, G. Magenes, M.C. Griffith, Dry stone masonry walls in bending Part I: static tests, International Journal of Architectural Heritage, 8, 1-28, 2014.

[15] M.C. Ferris, F. Tin-Loi, Limit analysis of frictional block assemblies as a mathematical program with complementarity constraints. International Journal of Mechanical Sciences, 43, 209-224, 2001.

[16] P. Trovalusci, R. Masiani, Non-linear micropolar and classical continua for anisotropic discontinuous materials. International Journal of Solids and Structures, 40, 1281-1297, 2003.

[17] C. Baggio, P. Trovalusci, Discrete models for jointed block masonry walls. The Sixth North American Masonry Conference, Philadelphia, Pennsylvania, June 6-9, 1993.

[18] J.B. Rondelet, Traité théorique et pratique de l'art de bâtir. Paris, France, 1802. 